\title{
Artesunate compared with quinine for the treatment of severe malaria in adult patients managed in an intensive care unit: A retrospective observational study
}

R M Mathiba, ${ }^{1}$ MB ChB, FCP (SA), MMed (Int Med); L R Mathivha, ${ }^{2,3}$ MB ChB, FCPaed (SA), DBS (BM), PGD (HSE); G D Nethathe, ${ }^{3} \mathrm{MB}$ ChB, DA (SA), FCA (SA), FANZCA, MMed (Anaes), Cert Crit Care (SA), PGD in HSE

${ }^{1}$ Department of Internal Medicine, Chris Hani Baragwanath Academic Hospital, Johannesburg, South Africa

${ }^{2}$ Department of Intensive Care, Chris Hani Baragwanath Academic Hospital, Johannesburg, South Africa

${ }^{3}$ Division of Critical Care, School of Clinical Medicine, University of the Witwatersrand, Johannesburg, South Africa; OrcID: 0000-0001-6875-8422

Corresponding author: R M Mathiba (rmathiba@ymail.co.za)

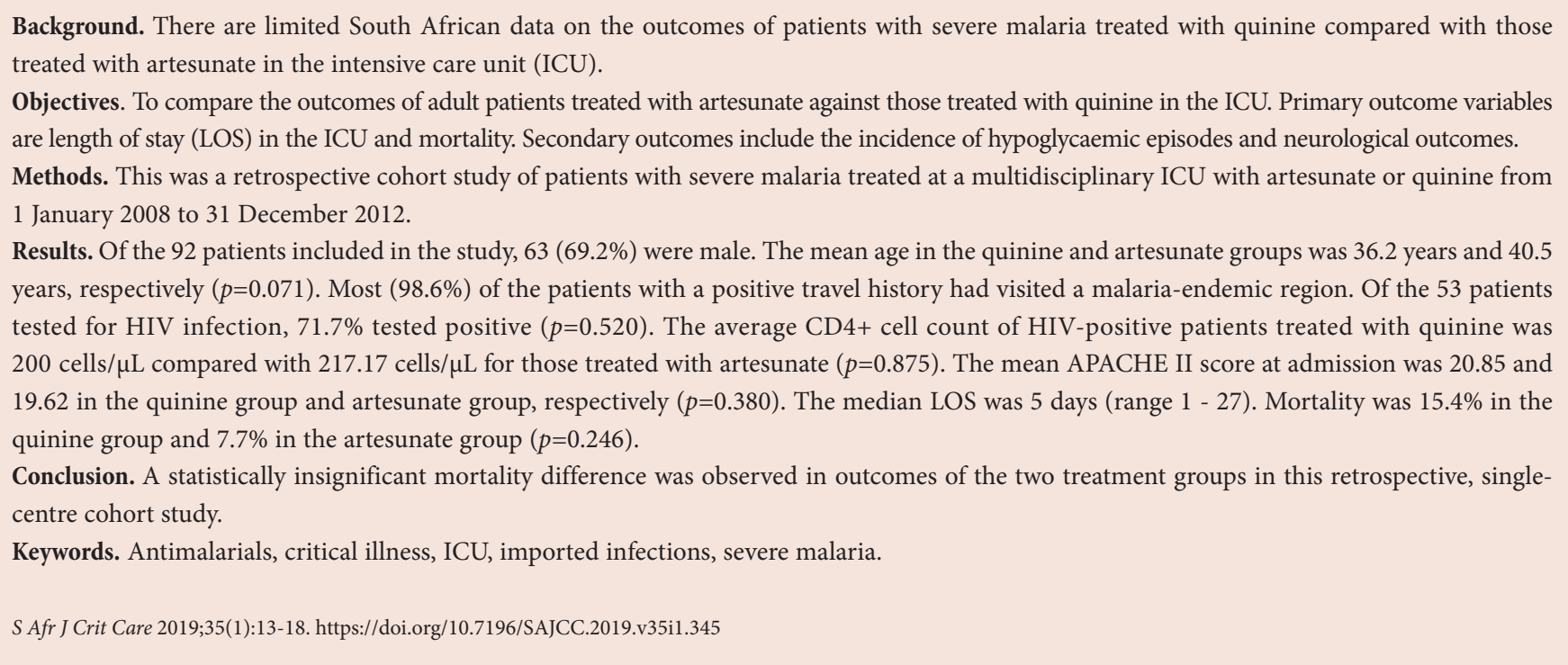

\section{Contribution of study}

- Intravenous artesunate is currently the preferred treatment in the management of patients with severe malaria. However, there are limited local data on the outcomes of artesunate v. quinine therapy for the management of severe malaria in highly monitored clinical environments in nonendemic regions of South Africa.

- We describe clinical characteristics, management and outcomes of patients with severe malaria treated with quinine and those treated with artesunate in the ICU in a non-endemic region.

Malaria is a preventable and treatable disease that is a major burden worldwide and especially in the African subregion. ${ }^{[1]}$ In 2012, about 207 million cases were reported worldwide, of which the majority were in the sub-Saharan region. ${ }^{[1]}$ Malaria is more prevalent in the tropics and, despite preventive measures, it remains a major reason for hospital admission in sub-Saharan countries. ${ }^{[2]}$ South African (SA) malaria transmission is seasonal and regional between October and May. ${ }^{[3,4]}$

In settings outside the intensive care unit (ICU), studies have shown a mortality benefit of artesunate over quinine. ${ }^{[2,5]}$ The South East Asian Quinine Artesunate Malaria Trial (SEAQUAMAT) was an open-label, randomised trial of 1461 patients with severe malaria. ${ }^{[5]}$ The mortality in the artesunate group was $15 \%$ compared with $22 \%$ in the quinine group $(p=0.000)$.

The African Quinine Artesunate Malaria Treatment (AQUAMAT) trial was performed in a paediatric population. It was the second biggest trial of the use of artesunate v. quinine in the treatment of severe Plasmodium falciparum malaria and was an open-label, randomised trial conducted in malaria-endemic regions in nine African countries. In-hospital mortality, the primary outcome, was $8.5 \%$ in the artesunate group compared with $10.9 \%$ in the quinine group $(p=0.002){ }^{[2]}$ The overall reduction in mortality in the AQUAMAT study was $22.5 \%$ $(p=0.002) .^{[2]}$ The results of these two trials prompted the World Health 
Organization (WHO) to change its guidelines and to advocate the use of artesunate in patients with severe malaria. ${ }^{[2]}$

Challenges relating to the availability of artesunate were initially a considerable barrier to its use in our hospital. Prior to its registration in SA in $2017,{ }^{[6]}$ artesunate was available only on a named-patient basis via the Medicines Control Council of South Africa through the Parenteral

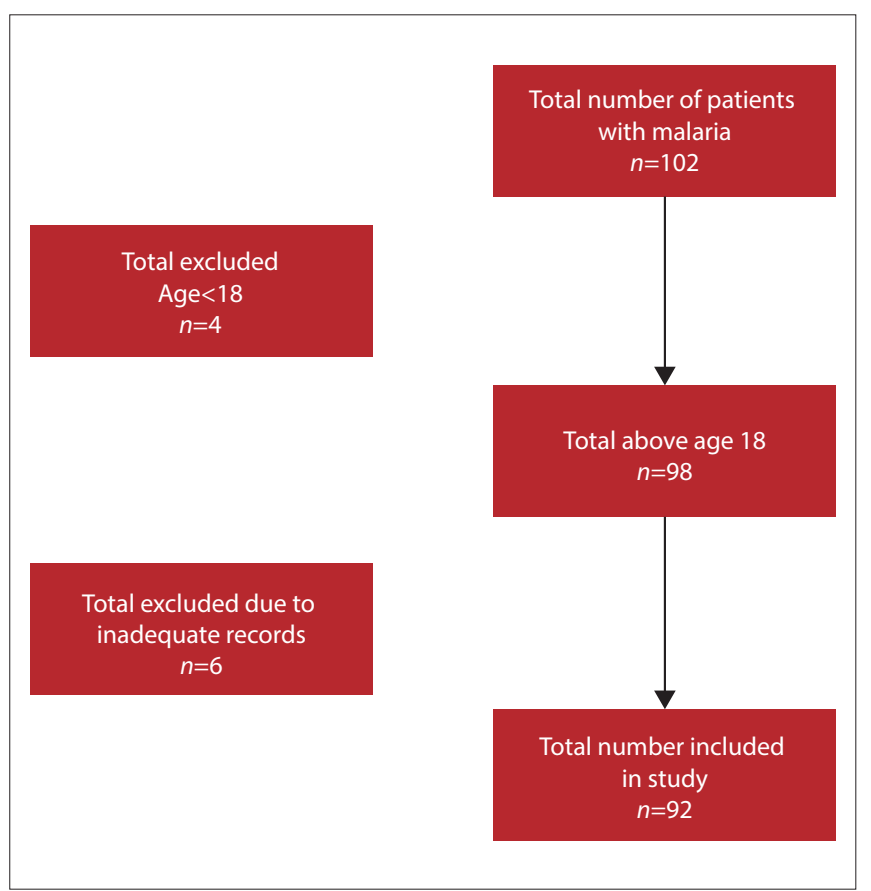

Fig. 1. Selection of study population.
Artesunate Access Programme, which was approved for patients with severe malaria and older than 12 years in $2009 .{ }^{[7]}$

There is a paucity of SA data regarding the outcomes of patients with severe malaria treated with quinine compared with those treated with artesunate in the ICU setting. SA is noted as a low-transmission area, although malaria is endemic in three provinces (Mpumalanga, KwaZuluNatal and Limpopo). ${ }^{[3,4]}$ Gauteng, the region in which our study is set, is a non-endemic area. Furthermore, the two large aforementioned trials (AQUAMAT and SEAQUAMAT) did not include patients managed in the ICU, where close monitoring and one-to-one nursing is standard. ${ }^{[2,5]}$ Close monitoring in an ICU setting allows early detection and management of potential complications of the disease or therapy. To date, only two relevant studies have been conducted in SA, and both focused only on prognostic indicators in a non-endemic area. ${ }^{[8,9]}$

The aim of the present retrospective review was to compare, over a 5-year period, outcomes of patients treated with artesunate with those treated with quinine at a multidisciplinary adult ICU of a large academic hospital. The research objectives were to assess and compare the severity of illness of patients in the two groups, as measured by APACHE II scores, and to compare admission parasite counts and laboratory data such as creatinine, lactate, bicarbonate and haemoglobin levels of patients on the respective therapies. In addition, ICU organ support interventions, ventilation, dialysis and inotropic support were compared for patients on quinine therapy and those treated with artesunate.

The primary outcomes were the length of ICU stay and mortality. Secondary outcomes included a change in neurological status and the incidence of hypoglycaemic episodes (defined as a glucose level $<2.2 \mathrm{mmol} / \mathrm{L}$ )

Table 1. Demographic characteristics of study population

\begin{tabular}{lllll}
\hline Variables & All, $\boldsymbol{n}(\%)$ & Quinine, $\boldsymbol{n}(\%)$ & Artesunate, $\boldsymbol{n}(\%)$ & $\boldsymbol{p}$-value \\
\hline Gender $(N=91)^{*}$ & - & $39(43.9)$ & $52(57.1)$ & 0.646 \\
$\quad$ Female & $28(30.8)$ & $13(33.3)$ & $15(28.8)$ & \\
$\quad$ Male & $63(69.2)$ & $26(66.7)$ & $37(71.2)$ & 0.071 \\
Age (years), mean $(N=91)^{*}$ & 38.6 & 36.2 & 40.5 & 1.000 \\
Positive travel history $(N=70)^{*}$ & - & $29(41.4)$ & $41(58.6)$ & \\
$\quad$ Malaria-endemic area & $69(98.6)$ & $29(100)$ & $40(97.6)$ & $1(2.4)$ \\
$\quad$ Non-endemic area & $1(1.4)$ & $0(0)$ & $36(67.9)$ & 0.520 \\
HIV status $(N=53)^{*}$ & - & $17(32.1)$ & $27(75)$ & \\
Positive & $38(71.7)$ & $11(64.7)$ & $9(25)$ & \\
Negative & $15(28.3)$ & $6(35.5)$ & &
\end{tabular}

Table 2. Investigations and clinical parameters recorded during patient admission

\begin{tabular}{|c|c|c|c|c|c|}
\hline \multirow[b]{2}{*}{ Variables } & \multirow[b]{2}{*}{ All $(N=92)^{*}$} & \multicolumn{2}{|c|}{ Treatment } & \multirow[b]{2}{*}{$t$-statistic } & \multirow{2}{*}{$\begin{array}{l}p \text {-value } \\
\text { (two-tailed) }\end{array}$} \\
\hline & & Quinine & Artesunate & & \\
\hline CD $4+$ count $($ cells $/ \mu \mathrm{L})(N=15)^{\dagger}$ & 213.73 & 200.00 & 217.17 & -0.161 & 0.875 \\
\hline Glasgow coma score at admission & 12.10 & 11.65 & 12.44 & -1.039 & 0.302 \\
\hline Parasite count $(\%)(N=75)^{\dagger}$ & $13.91 \%$ & $12.57 \%$ & $14.90 \%$ & -0.818 & 0.416 \\
\hline Lactate level $(\mathrm{mmol} / \mathrm{L}),(N=85)^{\dagger}$ & 4.76 & 5.23 & 4.37 & 1.066 & 0.291 \\
\hline Bicarbonate level (mmol/L) & 17.93 & 17.20 & 18.49 & -1.179 & 0.241 \\
\hline Haemoglobin level (g/dL) & 8.75 & 8.47 & 8.96 & -0.801 & 0.425 \\
\hline Blood glucose level $(\mathrm{mmol} / \mathrm{L})(N=87)^{\dagger}$ & 6.09 & 5.69 & 6.41 & -0.531 & 0.596 \\
\hline Creatinine level $(\mu \mathrm{mol} / \mathrm{L})$ & 351.04 & 300.35 & 390.04 & -1.329 & 0.187 \\
\hline APACHE II score & 20.15 & 20.85 & 19.62 & 0.882 & 0.380 \\
\hline
\end{tabular}




\section{Methods}

The study was conducted at a multidisciplinary adult ICU of a large academic hospital situated in an area of low malaria transmission. Both surgical and medical patients are admitted to the unit and referrals come mainly from the local community and peripheral hospitals in the surrounding towns and provinces. Ethical approval was obtained from the Human Research Ethics Committee (Medical) of the University of the Witwatersrand prior to commencement of the study (ref no. M150120).

\section{Study population and data collection}

This was a retrospective cohort study of adult patients with severe malaria treated with artesunate or quinine in the ICU. The study sample included all adult patients ( $\geq 18$ years) who were admitted to the ICU with a diagnosis of severe malaria between 1 January 2008 and 31 December 2012 and whose files could be found in our records. The diagnosis of severe malaria was based on WHO guidelines. There were no exclusion criteria.

Drug selection was influenced by availability. Quinine had been the therapeutic option of choice for the management of malaria in our unit prior to December 2009. Artesunate subsequently became available in our unit on a named-patient basis following the approval of the Parenteral Artesunate Access Programme earlier in 2009. Quinine was used only if artesunate was not available (out of stock).

Details of all identified patients were obtained from ICU records and entered into a password-protected spreadsheet. Fig. 1 further illustrates the sampling process.

\section{Statistical analysis}

Data were captured in a spreadsheet and then exported to SPSS (version 22) for statistical analysis. The analysis was based on the valid cases in the data file and excluded missing values.

Descriptive statistics such as frequency distributions were used to summarise categorical variables (e.g. drug type), whereas means and standard deviations were calculated to summarise numerical variables. A chi-squared test of association was used to assess whether there was an association between two categorical variables (e.g. drug type and comorbidities). A $p$-value $<0.05$ indicated a statistically significant association. An independent-samples $t$-test was used to compare the mean values of numerical variables between two groups. A significance level of $p<0.05$ was used.

Table 3. Association between administered drug and therapeutic intervention, and differences between treatment groups

\begin{tabular}{|c|c|c|c|c|c|}
\hline Intervention variable & $\begin{array}{l}\text { All }(N=92), \\
n(\%)\end{array}$ & $\begin{array}{l}\text { Quinine }(N=40) \text {, } \\
n(\%)\end{array}$ & $\begin{array}{l}\text { Artesunate }(N=52), \\
n(\%)\end{array}$ & $t$-statistic & $p$-value \\
\hline \multicolumn{6}{|l|}{ Type } \\
\hline Inotropic support & $57(62.0)$ & $27(67.5)$ & $30(57.7)$ & $\mathrm{n} / \mathrm{a}$ & 0.337 \\
\hline Renal replacement therapy & $52(56.5)$ & $21(52.5)$ & $31(59.6)$ & $\mathrm{n} / \mathrm{a}$ & 0.495 \\
\hline Mechanical ventilation & $34(37.0)$ & $20(50.0)$ & $14(26.9)$ & $\mathrm{n} / \mathrm{a}$ & 0.023 \\
\hline \multicolumn{6}{|l|}{ Duration (days), average } \\
\hline Inotropic support $(N=35)^{\star}$ & 3.91 & 4.62 & 3.50 & 0.812 & 0.423 \\
\hline Renal replacement therapy $(N=51)^{*}$ & 6.37 & 6.05 & 6.60 & -0.347 & 0.730 \\
\hline Mechanical ventilation $(N=34)^{*}$ & 5.74 & 5.55 & 6.00 & -0.24 & 0.812 \\
\hline
\end{tabular}

Table 4A. Primary outcomes as measured in the two treatment groups

\begin{tabular}{|c|c|c|c|c|}
\hline Primary outcome & Total $(N=92), n(\%)$ & Quinine $(N=40), n(\%)$ & Artesunate $(N=52), n(\%)$ & $p$-value \\
\hline Length of stay (days), median (range) & $5(1-27)$ & - & - & - \\
\hline$<5$ days & $42(45.7)$ & $18(45.0)$ & $24(46.2)$ & 0.738 \\
\hline 5 days & $9(9.8)$ & $5(12.5)$ & $4(7.7)$ & \\
\hline$>5$ days & $41(44.6)$ & $17(42.5)$ & $24(46.2)$ & \\
\hline Discharge & $81(89.0)^{*}$ & $33(84.6)^{\dagger}$ & $48(92.3)$ & 0.246 \\
\hline Death & $10(11.0)^{*}$ & $6(15.4)^{\dagger}$ & $4(7.7)$ & \\
\hline
\end{tabular}

Table 4B. Secondary outcomes as measured in the two treatment groups

\begin{tabular}{|c|c|c|c|c|}
\hline Secondary outcome & Total $(N=22), n(\%)$ & Quinine $(n=13), n(\%)$ & Artesunate $(n=9), n(\%)$ & $p$-value \\
\hline Complications & & & & 0.301 \\
\hline Seizures & $11(50.0)$ & $7(53.8)$ & $4(44.4)$ & \\
\hline Drowsiness & $4(18.2)$ & $3(23.1)$ & $1(11.1)$ & \\
\hline Pulmonary oedema & $2(9.1)$ & $0(0.0)$ & $2(22.2)$ & \\
\hline Vision loss & $1(4.5)$ & $1(7.7)$ & $0(0.0)$ & \\
\hline Speech defect & $1(4.5)$ & $0(0.0)$ & $1(11.1)$ & \\
\hline Psychosis & $1(4.5)$ & $1(7.7)$ & $0(0.0)$ & \\
\hline Confusion & $1(4.5)$ & $0(0.0)$ & $1(11.1)$ & \\
\hline Hypoglycaemic episodes & $1(4.5)$ & $1(7.7)$ & $0(0.0)$ & \\
\hline Glasgow coma score at discharge $(N=92)$ & 14.47 & 14.91 & 14.16 & 0.081 \\
\hline
\end{tabular}




\section{Results \\ Demographics}

The sample consisted of 92 patients, of whom 63 (69.2\%) were male (Table 1). Of the 92 patients, $43.9 \%(n=39)$ received quinine while $57.1 \%$ $(n=52)$ received artesunate. The mean age of patients in the quinine group was 36.2 years compared with 40.5 years in the artesunate group $(p=0.071)$. Of the patients who had a positive travel history, 69 (98.6\%) had travelled to a malaria-endemic region prior to admission. Of the 53 patients who were tested for HIV, 38 (71.7\%) tested positive while 15 (28.3\%) tested negative $(p=0.520)$.

\section{Physiological parameters}

Physiological parameters recorded during patient admission are shown in Table 2. For HIV-positive patients treated with quinine, the average CD4+ count was 200 cells $/ \mu \mathrm{L}$, compared with 217.17 cells $/ \mu \mathrm{L}$ for those treated with artesunate $(p=0.875)$. For patients treated with quinine, the mean parasite count on admission was $12.57 \%$ compared with $14.90 \%$ for patients treated with artesunate $(p=0.416)$. There were no significant differences between the two treatment groups with regard to mean bicarbonate $(p=0.241)$, lactate $(p=0.291)$, haemoglobin $(p=0.425)$ or creatinine levels $(p=0.187)$. The APACHE II score on admission was similar in the two groups $(p=0.380)$. The average blood glucose level was $5.69 \mathrm{mmol} / \mathrm{L}$ for patients treated with quinine and $6.41 \mathrm{mmol} / \mathrm{L}$ for patients treated with artesunate $(p=0.596)$.

\section{Therapeutic interventions}

Patients treated with quinine required inotropic support for an average of 4.62 days compared with 3.50 days for those treated with artesunate $(p=0.423$ ) (Table 3). There was no statistical difference with regard to the duration of renal replacement therapy between patients treated with quinine or artesunate ( 6.05 days v. 6.60 days; $p=0.730$ ) or with regard to mechanical ventilation between the two treatment groups (5.50 days v. 6.00 days; $p=0.812$ ).

\section{Outcomes}

The primary outcomes measured in this study were length of ICU stay and mortality (Table 4A).

The median length of ICU stay was 5 days (range 1 - 27) across the total sample. There was no statistically significant difference with regard to length of stay ( $p=0.738$ ) or mortality $(p=0.246$ ) between the two treatment groups. Of the patients admitted for fewer than 5 days, 18 (45.0\%) were treated with quinine, whereas $24(46.2 \%)$ were treated with artesunate. Of the patients admitted for more than 5 days, 17 (42.5\%) and 24 (46.2\%) were treated with quinine and artesunate, respectively.

There was no significant association between mortality and the treatment drug $(p=0.246)$. In total, 10 patients died in the ICU $(11.0 \%)$; a total of $89 \%$ of patients survived. Of the patients who were treated with quinine, 33 (84.6\%) were discharged alive. A similarly large percentage of patients were discharged alive from the artesunate treatment group $(n / N=48 / 52 ; 92.3 \%)$.

Secondary outcomes are described in Table 4B. In patients treated with quinine, the mean Glasgow coma score at discharge was 14.91 compared with 14.16 for patients treated with artesunate $(p=0.081)$. Seizures were the most commonly recorded neurological complication $(n / N=11 / 22)$. Only one hypoglycaemic event was recorded (from the quinine treatment group).

\section{Discussion}

Malaria is a major cause of death in sub-Saharan Africa. SA, and specifically the Gauteng province, is considered a non-endemic region. ${ }^{[4]}$
However, an increasing number of cases of severe malaria are seen, mostly among visitors from endemic areas entering the country. ${ }^{[4]}$ Of the 92 patients admitted to our ICU, just over two-thirds were male, which may reflect the gender differences in travel-associated disease described previously. ${ }^{[10]}$ Johannesburg has a large male migrant population, which may explain the higher prevalence of the disease in men. This is in contrast to other African regions were women and children are more likely to be affected than men. ${ }^{[1]}$

In addition, during the study period, SA had the highest HIV infection rate of any country in Africa, ${ }^{[3]}$ increasing patients' susceptibility to developing severe malaria as a result of immunosuppression. A higher HIV prevalence increases mortality from malaria. More than half of the patients tested for HIV infection in our study were positive (Table 1). As HIV and malaria infection follow the same geographical distribution, HIV-associated immunosuppression has been postulated to increase the risk of contracting malaria and reducing the efficacy of antimalarial therapy. ${ }^{[12]}$ In the AQUAMAT trial, high mortality was observed in the $6 \%$ of the population co-infected with HIV. The full extent of the pharmacokinetic interactions between antimalarial drugs and antiretrovirals used in the management of HIV is not well understood owing to limited published data. Difficulty in distinguishing between adverse reactions due to antimalarial therapy, antiretroviral therapy or severe malaria infection itself may pose additional diagnostic challenges. The increased incidence of severe malaria in HIV-infected patients is likely to add to the overall burden of disease, increasing morbidity and mortality in sub-Saharan African populations. ${ }^{[13]}$

The cost of parenteral artesunate is currently higher than that of quinine; however, when considering the associated reduction in mortality and shorter duration of treatment, artesunate becomes a cost-effective option. ${ }^{[14]}$ Access to artesunate has only recently become available (2017) in SA. ${ }^{[6]}$ Prior to this, artesunate was available to patients 12 years and older with severe malaria only on a named-patient basis via the Medicines Control Council of South Africa through the Parenteral Artesunate Access Programme. ${ }^{[7]}$

Several large studies have shown that artesunate is superior to quinine in the treatment of severe malaria. ${ }^{[2]}$ None of the studies comparing the two drugs was performed in a non-endemic ICU setting. Although SA is a non-endemic region and has a low transmission rate, the case fatality rate $(0.76 \%)$ has remained unchanged in over a decade, which is $0.26 \%$ higher than the WHO target of $0.5 \%{ }^{[14]}$

Reduced sensitivity to quinine is more common in Asia than in Africa. ${ }^{[15]}$ The emergence of artesunate resistance in South-East Asia has now also been reported in a number of studies and is characterised by decreased parasite clearance times. ${ }^{[16,17]}$ The pfkelch 13 mutation is commonly associated with artemisinin resistance seen in P. falciparum malaria in Asia, but some additional mutations seem to be emerging also from Africa, as recently demonstrated. ${ }^{[18-20]}$ Although resistance was not a focus of our study, the comments highlight the importance of continued access to artesunate as therapeutic option.

The mortality benefit seen with artesunate was greater in South-East Asian populations than in Africa. ${ }^{[2,5]}$ This may be due to less quinine resistance in P. falciparum malaria in Africa. ${ }^{[2]}$ Our study showed no statistically significant difference in mortality between patients treated with artesunate or quinine $(p=0.246)$. Mortality among patients treated with quinine in our study (15.4\%) was considerably lower than that seen in a previous study in our unit (28.5\%). Reasons for this result are unclear, but increased awareness and thus earlier presentation may have been a contributing factor. In addition, although a high prevalence of HIV infection may have been expected to increase rather than reduce 
mortality associated with severe malaria, the use of antiretroviral therapy might reduce the morbidity of severe malaria in HIV-infected individuals. ${ }^{[2]}$ The current standard of care for the treatment of malaria is similar in HIV-positive and HIV-negative patients. Further research is needed to clarify the clinical implications of the concurrent use of antiretroviral and antimalarial therapy.

Other advantages of artesunate over quinine include ease of administration and a minimal side-effect profile. The main adverse effects reported since the initiation of the artesunate programme relate to renal failure and haemolysis. ${ }^{[14]}$ Quinine treatment is associated with a number of side-effects and requires dose adjustment in patients with renal impairment, a condition frequently encountered in patients with severe

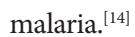

The ICU environment allows closer observation, earlier detection and detailed management of associated complications such as hypoglycaemia. Although the broader stage-specificity of artesuante over quinine is acknowledged, severe disease as would require ICU admission may negate the advantage when significant organ dysfunction has already set in. Artesunate may confer a mortality benefit in early disease and in patients who do not require interventions for organ support.

There was no difference in the length of stay between the different treatment modalities in our study. This is consistent with the results of the AQUAMAT trial, where the time to discharge was 3 days in both the artesunate and the quinine group $(p=0.059)$.

An open-label prospective randomised trial in a tertiary centre in India to compare the efficacy of quinine and artesunate in severe P. falciparum malaria is comparable to ours. ${ }^{[22]}$ Thirty-five patients who met WHO criteria for severe malaria were included. Of these, 18 received quinine and 17 received artesunate. The endpoints were parasite clearance time, fever clearance time, coma resolution time, adverse effects of the drugs and death. All patients were admitted to general wards, except four patients with acute respiratory distress syndrome, who were treated in the ICU. One patient died in each arm. Patients treated with artesunate experienced faster fever resolution and parasite clearance, whereas coma resolution was faster in the quinine group. As with our study, no mortality benefit was seen for artesunate over quinine. ${ }^{[22]}$

We did not find any statistically significant differences with regard to neurological outcomes between the two treatment groups, similar to reports also from other investigators. ${ }^{[23]}$ The finding is consistent with those from the AQUAMAT trial, where neurological sequelae were not found to be significantly different between patients treated with artesunate v. quinine, although the development of convulsions and coma and deterioration in the Glasgow coma score seemed to be slightly higher in the quinine group. ${ }^{[2]}$ Further investigation to evaluate this observation is warranted.

Hypoglycaemia is a notable and treatable cause of death in cases of severe malaria and is of multifactorial origin. ${ }^{[24]}$ In a recent Cochrane review $^{[50]}$ that compared the use of artesunate and quinine in the treatment of severe malaria, treatment with artesunate was found to be associated with a significant reduction in mortality and hypoglycaemia and improved parasite clearance in patients with severe malaria, and fewer hypoglycaemic episodes were seen for artesunate compared with quinine. Only one hypoglycaemic event was recorded in our study (from the quinine group). Such episodes are more likely to be missed in a general-ward environment.

\section{Study limitations}

The small sample size from a single centre may not be representative of the national population of critically ill malaria patients. The retrospective study design also presents a limitation.

Owing to limited resources and beds in our unit, it is possible that some patients with severe malaria, who may have required ICU admission, were treated in general medical wards and thus would not have been included in the study.

Despite these limitations, our findings expand on the current understanding of the treatment of severe malaria in highly monitored environments in non-endemic regions.

\section{Conclusion}

In this retrospective, single-centre cohort study, we observed a statistically insignificant mortality difference in outcomes of patients who received artesunate compared with those who received quinine.

\section{Acknowledgements. None.}

Author contributions. RMM was responsible for study conceptualisation, data collection and writing the research proposal and manuscript. GDN helped to revise the protocol design and prepare the final manuscript. LRM was involved in final document review.

Funding. None.

Conflicts of interest. None.

1. World Health Organization. World Malaria Report 2013. Country profiles. Geneva: WHO, 2013. http://www.who.int/malaria/publications/world_malaria_report_2013/wmr2013_country_profiles. pdf (accessed 10 July 2019).

2. Dondorp AM, Fanello CI, Hendriksen IC, et al. Artesunate versus quinine in the treatment of severe falciparum malaria in African children (AQUAMAT): An open-label, randomised trial. Lancet 2010;376(9753):1647-1657. https://doi.org/10.1016/S0140-6736(10)61924-1

3. National Department of Health. Malaria. Pretoria: NDOH, 2015.

4. Blumberg L, Frean J. Malaria control in South Africa - challenges and successes. S Afr Med J 2007;97(11):1193-1197.

5. Dondorp A, Nosten F, Stepniewska K, et al. Artesunate versus quinine for treatment of severe S0140-6736(05)67176-0

6. University of Cape Town Medicines Information Centre. Artesunate now registered in South Africa. http://www.mic.uct.ac.za/news/artesunate-now-registered-sa (accessed 10 July 2019).

7. Visser Kift E, Kredo T, Barnes KI. Parenteral artesunate access programme aims at reducing malaria fatality rates in South Africa. S Afr Med J 2011;101(4):240-241. https://doi.org/10.7196/samj.4761

8. Bruwer JW, Koegelenberg CFN, Lalla U, Irusen E. Malaria in the intensive care unit of a tertiary hospital in a non-endemic area of South Africa. A nine-year retrospective descriptive study. S Afr Respir J 2009;19(1):6-8.

9. Blumberg L, Lee RP, Lipman J, Beards S. Predictors of mortality in severe malaria: A twoyear experience in a non-endemic area. Anaesth Intensive Care 1996;24(2):217-223. https://doi. org/10.1177/0310057x9602400213

10. Schlagenhauf $\mathrm{P}$, Chen LH, Wilson ME, et al. Sex and gender differences in travel-associated disease. Clin Infect Dis 2010;50(6):826-832. https://doi.org/10.1086/650575

11. Heggenhougen, HK, Hackethal V, Vivek P, United Nations Development Program/World Bank/ World Health Organization Special Programme for Research and Training in Tropical Diseases. The behavioural and social aspects of malaria and its control: An introduction and annotated bibliography. Geneva: WHO, 2003. http://www.who.int/iris/handle/10665/42504 (accessed 6 October 2018).

12. Lu F, Culleton R, Zhang M, et al. Emergence of indigenous artemisinin-resistant Plasmodium falciparum in Africa. N Engl J Med 2017;376(10):991-993. https://doi.org/10.1056/nejmc1612765

13. González R, Ataíde R, Naniche D, Menéndez C, Mayor A. HIV and malaria interactions: Where do we stand? Expert Rev Anti Infect Ther 2012;10(2):153-165. https://doi.org/10.1586/eri.11.167

14. Department of Health (South Africa). National Essential Medicine List Medication Review Process. Primary HealthCare. Component: Malaria. http://www.health.gov.za/category/285/phc (accessed 10 July 2019).

15. Achan J, Talisuna AO, Erhart A, et al. Quinine, an old anti-malarial drug in a modern world: Role in the treatment of malaria. Malar J 2011;10:144. https://doi.org/10.1186/1475-2875-10-144

16. Ferreira PE, Culleton R, Gil JP, Meshnick SR. Artemisinin resistance in Plasmodium falciparum What is it really? Trends Parasitol 2013;29(7):318-320. https://doi.org/10.1016/j.pt.2013.05.002

17. Dondorp AM, Nosten F, Yi P, et al. Artemisinin resistance in Plasmodium falciparum malaria. N Engl J Med 2009;361(5):455-467. https://doi.org/10.1056/NEJMoa0808859

18. Ashley EA, Dhorda M, Fairhurst RM, et al. Spread of artemisinin resistance in Plasmodium falciparum malaria. N Engl J Med 2014;371(5):411-423. https://doi.org/10.1056/NEJMoa1314981

19. Imwong M, Suwannasin K, Kunasol C, et al. The spread of artemisinin-resistant Plasmodium falciparum in the Greater Mekong subregion: A molecular epidemiology observational study. Lancet Infect Dis 2017;17(5):491-497. https://doi.org/10.3410/f.727261093.793529467

20. Sutherland CJ, Lansdell P, Sanders M, et al. pfk13-Independent treatment failure in four imported cases of Plasmodium falciparum malaria given artemether-lumefantrine in the United Kingdom. Antimicrob Agents Chemother 2017;61(3):e02382-16. https://doi.org/10.1128/aac.02382-16 

21. Brentlinger PE, Behrens CB, Kublin JG. Challenges in the prevention, diagnosis, and treatment
of malaria in human immunodeficiency virus-infected adults in sub-Saharan Africa. Arch Intern Med 2007;167(17):1827-1836. https://doi.org/10.1001/archinte.167.17.1827

22. Haroon N, Amichandwala K, Solu MG. Comparative efficacy of quinine and artesunate in the treatment of severe malaria: A randomized control trial. JK Science 2005;7(1):1-4.

23. Jones KL, Donegan S, Lalloo DG. Artesunate versus quinine for treating severe malaria. Cochrane Database Syst Rev 2007;(4):CD005967. https://doi.org/10.1002/14651858.cd005967
24. Kochar DK, Thanvi I, Kumawat BL, Shubhakaran K, Agarwal N. Importance of blood glucose level at the time of admission in severe and complicated malaria. J Assoc Physicians India 1998;46(11):923-925.

Reviewed 19 January 2018. Accepted 8 October 2018 